\title{
Transvaginal/transrectal ultrasound-guided aspiration biopsy for diagnosis of pelvic/pelvic floor tumors in females: A retrospective analysis
}

\author{
CHUANFEN GAO ${ }^{1}$, LING WANG ${ }^{1}$, CHAOXUE ZHANG $^{1}$ and $\mathrm{XIN} \mathrm{LI}^{2}$ \\ ${ }^{1}$ Department of Ultrasound, The First Affiliated Hospital of Medical University of Anhui, Hefei, Anhui 230022; \\ ${ }^{2}$ Department of Interventional Ultrasound, Chinese PLA General Hospital, Beijing 100853, P.R. China
}

Received March 7, 2019; Accepted April 24, 2019

DOI: 10.3892/etm.2019.7563

\begin{abstract}
Ultrasound guidance for histological diagnosis is in real-time, convenient, and economical. The aim of this study was to determine whether transvaginal ultrasound (TVUS)and transrectal ultrasound (TRUS)-guided aspiration biopsy allows detection of a malignant pathology of pelvic/pelvic masses. Data of 40 patients with pelvic and pelvic mass lesions by computed tomography or magnetic resonance imaging underwent TVUS- or TRUS-guided biopsy. Tissue samples obtained were assessed on suitability for histopathologic evaluation. The post-biopsy complication was monitored. All the specimens in the pelvic floor, vaginal stump, vaginal fornix, cervix, and posterior wall of the anal canal were adequate for histologic diagnosis. There were no post-biopsy complications. Transvaginal/transrectal ultrasound-guided aspiration biopsy is safe and simple. It can be used for the diagnosis and differential diagnosis of pelvic and pelvic floor lesions in women. Prospective studies are needed to test diagnostic performance across clinical scenarios.
\end{abstract}

\section{Introduction}

Ultrasound-guided intervention has many benefits. The visually clear real-time pathway guarantees negligible radiation hazard, secures procedural safety for aspiration, biopsy, and ablation in the treatment of multiple organ diseases, enhances multi-dimensional capability, provides convenience, decreases procedure time, and minimizes cost (1,2). Also, the pre-operative sampling of tumor mass is suggested to confirm the presence of cancer and ascertain morphological subtype before neo-adjuvant chemotherapy may lead to the potential

Correspondence to: $\mathrm{Dr} \mathrm{Xin} \mathrm{Li}$, Department of Interventional Ultrasound, Chinese PLA General Hospital, 28 Fuxing Road, Beijing 100853, P.R. China

E-mail: lixin301@hotmail.com

Key words: aspiration biopsy, pelvic, transvaginal, transrectal, ultrasound-guided diagnostic misinterpretation of tumor cells and difficulties in detecting residual tumor after neoadjuvant chemotherapy of bulky malignant tissues (3).

Ultrasound-guided intervention has technically evolved with clinical procedures conducted at the abdomen, thorax and urogenital system (4). Transvaginal ultrasound (TVUS)guided biopsy is safe and effective in the diagnosis of pelvic lesions (5-15).

TVUS-guided gun biopsy of the uterus and ovaries in the office setting histologically confirmed 19 of the $22(86.4 \%)$ preliminary equivocal ultrasound diagnosis of adenomyosis, leiomyoma and benign ovarian mass (5). The diagnostic accuracy of TVUS using histopathology as a gold standard in identifying endometrial hyperplasia among 263 perimenopausal women presenting with abnormal uterine bleeding was found to be $75.6 \%$ (6).

TVUS has a high negative predictive value (99.1\%) for an endometrial thickness of $10.8 \mathrm{~mm}$ in the evaluation of 100 women with post-menopausal bleeding (7). The TVUS biopsy diagnosis of peritoneal carcinomatosis and recurrent pelvic malignancy was validated in a cohort of 50/54 (93\%) women by comparison with the histopathologic specimen or clinical course and outcome (8). TVUS-guided core needle biopsy adequately obtained tumor samples from 200 women with abdominopelvic or pelvic masses with 190 of $200(95.0 \%)$ verified before treatment (9). Of the 200, 97 (48.5\%) were inoperable tumors, $13(6.5 \%)$ were metastatic, 45 (22.5\%) were recurrent and $45(22.5 \%)$ were rare tumors (9). In 55 women with pelvic masses detected on computed tomography (CT) or magnetic resonance imaging (MRI) before the biopsy, $46(84 \%)$ of the pelvic samples from TVUS core biopsy were confirmed to be either malignant or benign, and $5(9 \%)$ were inflammatory lesions showing an overall diagnostic accuracy of 51/55 (93\%) (10). Of the 48 women diagnosed by ultrasound alone as having adenomyosis, 37 (77\%) were histologically confirmed as having adenomyosis after TVUSguided biopsy (11). Samples obtained by TVUS guided uterine core biopsy from 80 cases of pre-menopausal women scheduled for hysterectomy proved to be useful in the investigation of early pathogenesis of adenomyosis (12).

Transrectal ultrasound (TRUS)-guided core biopsy confirmed recurrent carcinoma of the uterine cervix in 
16/17 (94\%) of women with non-diagnostic vaginal cytology and transvaginal punch biopsy (13). TRUS-guided biopsy revealed recurrent pelvic malignancy in 5/8 (62\%) in women presenting with abdomino-pelvic and back pain (14). TRUS examination was assessed to have high diagnostic power for polycystic ovary syndrome among 183 Korean women aged 21-32 years (15).

Of the 11 studies that reported safety and efficacy, only one study reported vaginal bleeding, 10/55 (18\%) and gross hematuria, 2/55 (4\%) as TVUS procedure-related complications (10). There was no reported complication with TRUS-guided biopsy in any of the reports.

This study aimed to determine the accuracy and safety of transvaginal and transrectal core needle biopsy of pelvic cavity masses under ultrasound guidance. The authors undertook this study since there is scant literature on the histological findings from biopsies taken directly from the pelvic cavity and from pelvic floor lesions.

\section{Materials and methods}

Subjects. We randomly obtained medical records of female patients. A consecutive series of 40 patients with the diagnosis of pelvic or pelvic floor masses between July 2015 and August 2017 at the Department of Ultrasound of the First Affiliated Hospital of Medical University of Anhui (Hefei, China) were considered eligible and chosen for satisfying the inclusion criteria. Inclusion criteria were as follows: i) Mass was detected by MRI or positron emission tomography (PET)/CT one month prior to biopsy, ii) primary origin was undetermined; iii) mass was visible by either transvaginal or transrectal ultrasound technique, iv) mass was in proximity, v) accessible to either TVUS- or TRUS-guided biopsy for tissue sampling, and vi) histological confirmation is needed for further patient management. Exclusion criteria were as follows: i) No ultrasound-guided needle path, ii) poor coagulation function, iii) severe infection, and iv) severe heart and lung insufficiency.

Clinical laboratory indicators of patient status and risk of complication were evaluated. The laboratory indicators were complete blood count, prothrombin time, international normalized ratio, activated partial thromboplastin time. We retrospectively analyzed extracted clinicopathologic data.

Informed consent and ethics approval. Before the procedure, patients were informed of the risk of complications and potential damage to adjacent structures along the path of the needle. Consent to proceed with the biopsy was obtained before the procedure.

Before inclusion into the retrospective study, patients or legally authorized representatives of subjects were contacted. All the participants gave their informed consent for inclusion and use of patient information. The protocol of the study was approved by the Medical University of Anhui Institutional Review Board. The IRB approval project identification code is $\mathrm{AF} / \mathrm{SC}-08 / 02.0$. The study was conducted as per the Declaration of Helsinki.

Instrument and biopsy procedure. None of the included patients had contraindications for biopsy. Every biopsy was performed by one of two experienced physicians $(\mathrm{CG}, \mathrm{LW})$ who had worked at least five years at the interventional ultrasound department using US instrument (Logiq E9; GE Healthcare, Chicago, IL, USA). TVUS- and TRUS-guided biopsies were performed in the lithotomy position with empty bladder after sterilization of the vagina and anus (16). The procedure utilized a reusable automatic biopsy gun (Bard Biopsy, Tempe, AZ, USA) compatible with an 18 gauge $15 \mathrm{~cm}$ tru-cut needle. The needle was inserted parallel to the transvaginal US probe and was directed to the lesion with an attached needle guide.

Local anesthesia and conscious sedation were not used. The biopsy needle with ultrasonic dynamic monitoring led the passage avoiding bowel, blood vessels, and bladder. The morphological characteristics of the mass, size, location, relationship with the adjacent tissues, and proximity to the vagina or rectum were observed before puncture. Upon reaching the lesion edge and gaining a penetration depth of $>2.0 \mathrm{~cm}$ with strong echo lesions, biopsy specimens were drawn from different directions (17). All the specimens obtained were immediately placed on a sterile filter paper and fixed in $10 \%$ formaldehyde solution (Sigma-Aldrich; Merck KGaA, Darmstadt, Germany). Sections and slides from paraffin-embedded tissue blocks as samples were stained with hematoxylin-eosin.

After the transvaginal procedures, three sterilized cotton balls were immediately tucked into the biopsy sites for hemostasis. Bleeding and other possible complications were checked after the cotton balls were taken out after $30 \mathrm{~min}$. Patients were observed around $30 \mathrm{~min}$ to $1 \mathrm{~h}$ with frequent vital signs monitoring in a dedicated area. Safety of the procedure was concluded if there were no or minor complications. If without discomfort or complications, patients were returned to the ward or sent home. The procedure and diagnostic criteria of TRUS-guided biopsy were similar to that of TVUS (18).

Radiologic, pathologic, and clinical data analyses. All archived CT, MRI, and ultrasonography (US) images from the picture archiving and communication system (PathSpeed, GE Healthcare, Milwaukee, WI, USA) were re-evaluated. Radiologists were aware of the history of the patient illness but blinded to all other clinical information. Two radiologists independently evaluated the pelvic lesions based on the: i) lesion size, ii) lesion nature (e.g., solid or cystic) (19), and iii) lesion site. The biopsy core number was counted from images, and the biopsy distance was determined from the standard reports. The biopsy distance was defined by the measured mean length of the biopsy needle seen on US images. Disagreements of the evaluation of two radiologists were resolved by consensus. If no agreement was reached, a third evaluator was consulted for final consensus.

An experienced pathologist evaluated the histology of the specimen. The pathologist was aware of the history of the patient illness but blinded to all other clinical information including the imaging results (20). The step detects the presence or absence of malignancy thereby detecting or excluding the diagnosis (21). Possible complications based on the patients' medical records were evaluated using the Clavien-Dindo classification (22).

\section{Results}

Of the 40 female patients included in the study, 39 had TVUS, and 1 had TRUS-guided biopsy. The mean age was 54 years 
Table I. Normal values of coagulation in patients undergoing TVUS- and TRUS-guided aspiration biopsy.

\begin{tabular}{lcc}
\hline $\begin{array}{l}\text { Hematologic } \\
\text { test }\end{array}$ & $\begin{array}{c}\text { Normal range } \\
\text { of values (reference) }\end{array}$ & Cut-off \\
\hline $\begin{array}{lcc}\text { Activated partial } \\
\text { thromboplastin time }\end{array}$ & $28.0-42.0$ & 45 \\
INR & $0.85-1.15$ & 1.6 \\
Platelets, $10^{9} / 1$ & $125-350$ & 50 \\
Prothrombin time & $11.0-16.0$ & 18 \\
\hline
\end{tabular}

INR, international normalized ratio; TVUS, transvaginal ultrasound; TRUS, transrectal ultrasound.

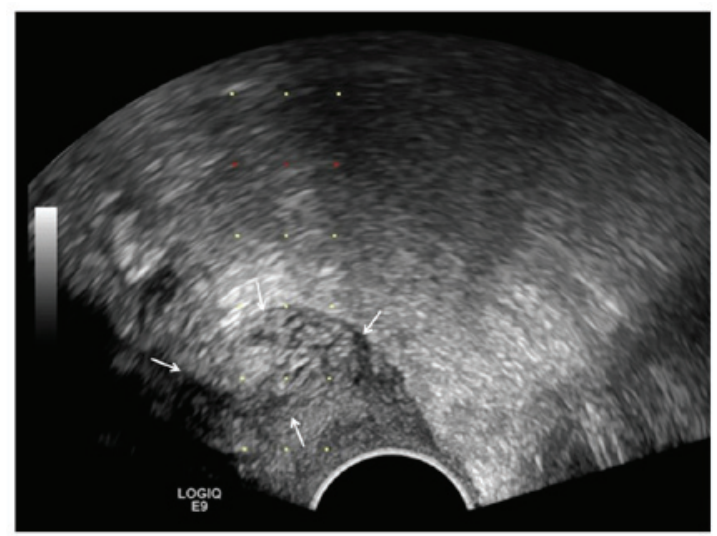

Figure 1. TVUS of a 67/F patient with endometrial cancer. Biopsy was taken at the vaginal stump, with a mass of $3.0 \times 1.7 \mathrm{~cm}$, indicated by the white arrows. TVUS, transvaginal ultrasound.

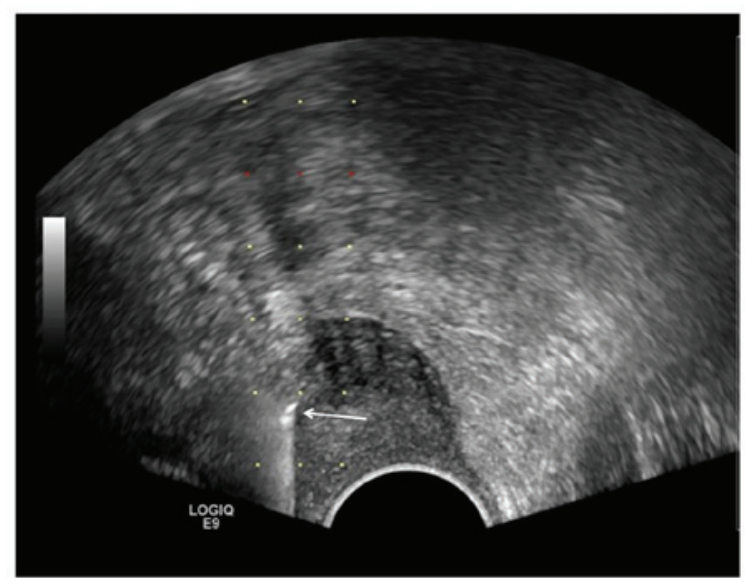

Figure 2. The puncture needle enters the lesion of the vaginal stump as shown by the white arrow.

(range, 46-69 years). All laboratory results were within the acceptable normal range of coagulation parameters (Table I). There were no complications identified.

The median lesion size was $5.5 \mathrm{~cm}$ (range, $1-15 \mathrm{~cm}$ ). Thirty-four of the lesions were solid while six were cystic. The mean distance of the biopsy was $2.4 \mathrm{~cm}$ (range, 1.4-5.6 cm). The median number of biopsy cores obtained from each

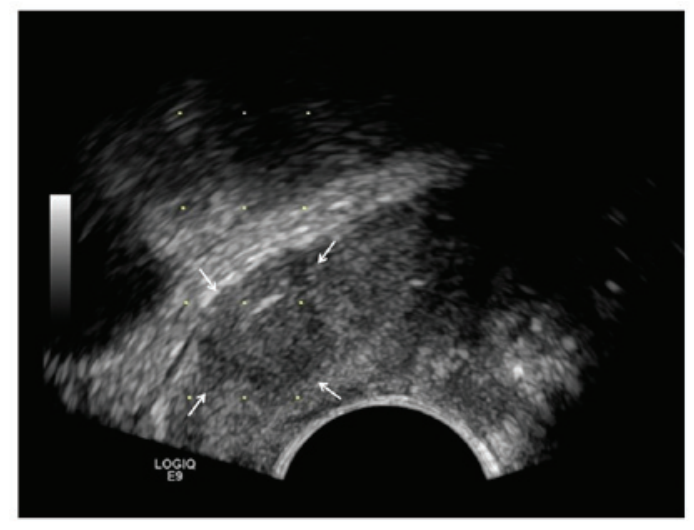

Figure 3. TVUS of a $62 / \mathrm{F}$ patient with cervical cancer. Biopsy was taken at the cervix. A $2.8 \times 2.3 \mathrm{~cm}$ mass is indicated by the white arrows. TVUS, transvaginal ultrasound.

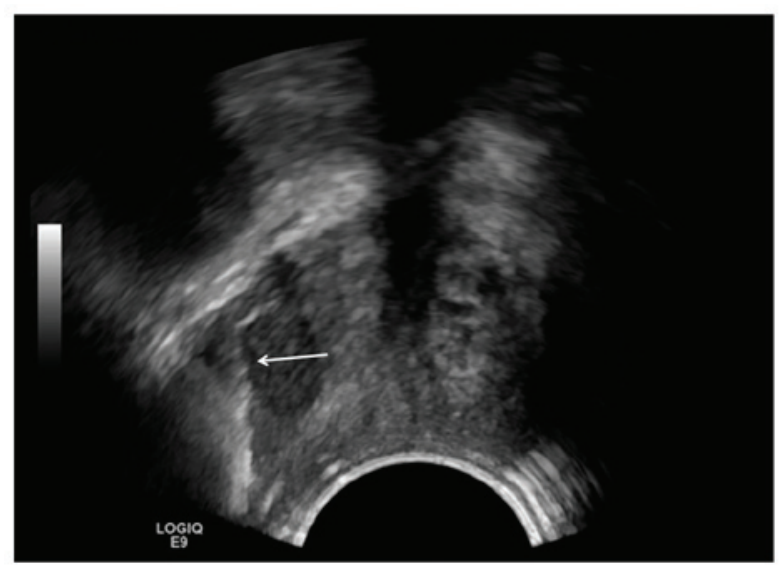

Figure 4 . The puncture needle enters the cervical lesion as shown by the white arrow.

patient was 4.0 (range, 2-7 cores). The specimens (Table II) were obtained from pelvic cavity and pelvic floor in 18 cases (45\%), the vaginal stump in 6 cases (15\%), the cervix in 2 cases (5\%) and the vaginal fornix in 13 cases (32.5\%). Representative cases are shown in Figs. 1-4.

All the specimens were adequate for histologic evaluation and diagnosis with $72 \%(28 / 39)$ being identified as primary and $13 \%(5 / 39)$ as metastatic malignancy. There was one diagnosed case of rectal cancer with a post-operative specimen from the posterior wall of the anal canal obtained by TRUSguided biopsy (2.5\%).

\section{Discussion}

A retrospective analysis was performed in our center to determine whether TVUS- and TRUS-guided aspiration biopsy allows the detection of a malignant pathology in the pelvic/pelvic floor. The study shows detection in almost all suspicious clinical cases by MRI or PET/CT consistent with a more than $90 \%$ adequacy and accuracy of other studies (8-10). An experienced pathologist provided the expertise that has optimized the use of aspiration biopsy.

TVUS- or TRUS-guided aspiration biopsy has several advantages. The biopsy is technically easy and does need 
Table II. Malignancy proven lesions from transvaginal ultrasound-guided aspiration biopsies.

\begin{tabular}{|c|c|c|}
\hline Patient & $\begin{array}{c}\text { Age } \\
\text { (years) }\end{array}$ & $\begin{array}{l}\text { MRI and PET/CT } \\
\text { findings at sites }\end{array}$ \\
\hline 1 & 84 & Pelvic cystic, solid mass \\
\hline 2 & 67 & Vaginal stump hypoechoic \\
\hline 3 & 60 & $\begin{array}{l}\text { Bottom of } \\
\text { the pelvic floor }\end{array}$ \\
\hline 4 & 52 & $\begin{array}{l}\text { Low echo above } \\
\text { the vaginal stump }\end{array}$ \\
\hline 5 & 46 & $\begin{array}{l}\text { Cervical anterior lip } \\
\text { hypoechoic }\end{array}$ \\
\hline 6 & 68 & Pelvic floor cervix \\
\hline 7 & 46 & $\begin{array}{l}\text { Low echo above } \\
\text { the vaginal stump }\end{array}$ \\
\hline 8 & 54 & $\begin{array}{l}\text { Right ovarian } \\
\text { solid lesion }\end{array}$ \\
\hline 9 & 47 & $\begin{array}{l}\text { Low echo } \\
\text { of the left uterus }\end{array}$ \\
\hline 10 & 62 & $\begin{array}{l}\text { Hypoechoic lesion on the left } \\
\text { side of the posterior vagina }\end{array}$ \\
\hline 11 & 46 & $\begin{array}{l}\text { Cervical anterior and posterior } \\
\text { lip hypoechoic, cervix } 1 \text {, } \\
\text { cervix } 2 \text {, anterior vaginal wall }\end{array}$ \\
\hline $12^{\mathrm{a}}$ & 47 & Cervical, ovary \\
\hline 14 & 50 & Hypoepitic lesions above the vagina \\
\hline 15 & 67 & Vaginal stump cystic, solid mass \\
\hline 16 & 60 & Right ovarian giant cystic \\
\hline
\end{tabular}

Double ovarian solid mass

Left attachment

Grayish white,

poorly differentiated cancer

Low-grade adenocarcinoma

Grayish white, poorly

differentiated adenocarcinoma

Grayish white, poorly differentiated

squamous cell carcinoma

Medium-differentiated adenocarcinoma, metastatic carcinoma of the upper digestive source may be large

Gray-white, poorly differentiated urothelial carcinoma may be large

Gray-white, poorly differentiated cancer, combined with a history of breast cancer metastasis may be large

Grayish white, spindle cell lesion, no excluding sex stromal tumor

3 grayish white, high-grade serous carcinoma, source of female reproductive system

3 grayish white, ovarian serous carcinoma metastasis

High-grade squamous intraepithelial

neoplasia CIN3, suspicious microinvasive, Cervical cancer chronic inflammation of vaginal lesions

1 anterior lip of the cervix, smooth muscle

1 posterior lip of the cervix, adenocarcinoma;

2 ovarian cancer, adenocarcinoma;

Grayish white 3, leiomyoma

Poorly differentiated cancer

Medium differentiated

adenocarcinoma

Poorly differentiated cancer

Poorly differentiated cancer

Squamous cell carcinoma

Metastatic poorly

differentiated adenocarcinoma

Poorly differentiated cancer

Poorly differentiated cancer

Serous carcinoma

Endometriosis

Spindle cell tumor

Leiomyoma

Serous carcinoma

Adenoid cystic carcinoma

Adenocarcinoma

Poorly differentiated

cancer
Ovarian cancer

Ovarian cancer

Ovarian cancer

Cervical cancer

Metastatic adenocarcinoma, upper gastrointestinal Cervical cancer

Metastatic poorly differentiated, breast

Ovarian cancer

Ovarian cancer

Ovarian cancer

Straight B

junction tumor ovarian metastasis

Uterine fibroids

Ovarian cancer

Metastatic non-small cell lung cancer, adenocarcinoma

Ovarian cancer

Ovarian cancer

Cervical cancer

Metastatic non-small cell lung cancer, adenocarcinoma

Cervical cancer

Cervical cancer

Ovarian cancer

Endometriosis

Sex cord stromal tumor

Vaginal leiomyoma

Ovarian cancer

Ovarian cancer

Endometrial cancer

Ovarian cancer 
Table II. Continued.

\begin{tabular}{|c|c|c|c|c|}
\hline Patient & $\begin{array}{c}\text { Age } \\
\text { (years) }\end{array}$ & $\begin{array}{l}\text { MRI and PET/CT } \\
\text { findings at sites }\end{array}$ & $\begin{array}{l}\text { Pathomorphological } \\
\text { findings }\end{array}$ & Diagnosis \\
\hline 31 & 52 & Posterior wall of the lower vagina & Leiomyoma & Uterine fibroids \\
\hline 32 & 64 & Above the vaginal stump & Endometrial stromal sarcoma & Uterine cancer \\
\hline 33 & 70 & Cervical hypoechoic & Inflammatory & Inflammatory cells, cervix \\
\hline 34 & 59 & Uterine rectal fossa lesion & Serous carcinoma & Ovarian cancer \\
\hline 35 & 42 & Left echo low echo nodule & Metastatic cancer & Endometrial cancer \\
\hline 36 & 53 & Uterine rectal fossa lesion & Serous carcinoma & Ovarian cancer \\
\hline 37 & 46 & Subcutaneous hypoechoic & Adenoid cystic carcinoma infiltration & Vaginal cancer \\
\hline 38 & 42 & Pelvic mass & Low-grade adenocarcinoma & Cervical cancer \\
\hline 39 & 41 & Vaginal stump & Squamous cell carcinoma & Cervical cancer \\
\hline 40 & 54 & Uterine rectal fossa & Left ovarian granuloma & Ovarian cancer \\
\hline
\end{tabular}

${ }^{a}$ Laparoscopy and transvaginal ultrasound-guided. CT, computed tomography; MRI, magnetic resonance imaging; PET, positron emission tomography.

anesthesia. Either of the two techniques can be used as a first-line investigation in the evaluation of women with pelvic or pelvic floor masses $(6,9)$. The biopsy can be supplemented with procedures such as laparoscopy for lesions involving ovary and cervix as experienced in the hospital. In addition to the traditional benefits of ultrasound-guided biopsy $(1,2)$, seeding by malignant cells seems low risk (9). Also, it is not known whether the risk of infection is high with the transrectal or transvaginal approaches. Antibiotic prophylaxis is well-established in transrectal biopsy of the prostate (23). However, whether infection and subsequent antibiotic prophylaxis are necessary for TVUS-guided biopsy remains to be observed.

The present study has several limitations. As a retrospective analysis using medical chart reviews, data inputted from medical records were not sufficient to determine diagnostic performance. Analysis to provide valid and reliable diagnostic performances (sensitivity, specificity, positive predictive value, negative predictive value, accuracy) requires the identification of a set of variables to provide the best prediction. Missing data precluded the detection of increasing diagnostic certainty from imaging to histopathology. Detection or exclusion of malignancy using test-result based sampling or case-referent sampling is ideal (21).

Second, the diagnostic test is almost always not applied in isolation but in combination (21). Interacting variables (relevant clinical variables, tumor markers, CT/MRI, clinical stage of cancer) increase sample size and may not be easy to be obtained. To conduct this kind of study, we may have to collaborate with other hospital centers. Finally, there were case scenarios in the management of cancer patients that were not encountered but are potentially significant: i) A previously diagnosed benign tumor turning out to be malignant, ii) distinguishing either a recurrence or post-treatment fibrosis, iii) whether TRUS complements TVUS, and iv) follow-up stage of cancer management.

Despite the limitations, the validity of the pathomorphologic findings and final diagnosis were not compromised because the pathologist was blinded to all other clinical information including the imaging results.
Ultrasound-guided transvaginal or transurethral biopsy seems to be a reliable and safe procedure for histopathological evaluation of the pelvic cavity and pelvic mass lesions. Prospective studies of adequate sample size are needed to evaluate the usefulness of the procedures across various clinical case scenarios.

\section{Acknowledgements}

Not applicable.

\section{Funding}

This study was supported by the National Scientific Foundation Committee of China (No. 81801723) and the Clinical Research Support Foundation of the Chinese PLA General Hospital (No. 2017FC-CXYY-3005).

\section{Availability of data and materials}

The datasets used and/or analyzed during the current study are available from the corresponding author on reasonable request.

\section{Authors' contributions}

CG. participated in the analysis and interpretation of data, and drafted the manuscript. XL made substantial contributions to the conception and design of the study. CG, LW and CZ carried out the study and collected the data. XL contributed to revising the manuscript critically for important intellectual content. All authors read and approved the final manuscript.

\section{Ethics approval and consent to participate}

This retrospective medical record review was approved by the Institutional Review Board/ Clinical Medical Research Ethics Committee of the First Affiliated Hospital of Anhui Medical University (Approval no: AF/SC-08/02.0) 


\section{Patient consent for publication}

Not applicable

\section{Competing interests}

The authors declare that they have no competing interests.

\section{References}

1. Copelan A, Scola D, Roy A and Nghiem HV: The myriad advantages of ultrasonography in image-guided interventions. Ultrasound Q 32: 247-257, 2016.

2. Park BK: Ultrasound-guided genitourinary interventions: Principles and techniques. Ultrasonography 36: 336-348, 2017.

3. McCluggage WG, Lyness RW, Atkinson RJ, Dobbs SP, Harley I, McClelland HR and Price JH: Morphological effects of chemotherapy on ovarian carcinoma. J Clin Pathol 55: 27-31, 2002.

4. Dietrich CF and Numberg E (eds): A Practical Guide and Atlas. In: Intervention Ultrasound: 1st edition. Thieme, Stuttgart, Germany, pp404, 2014

5. Walker $\mathbf{J}$ and Jones $\mathrm{K}$ : Transvaginal ultrasound guided biopsies in the diagnosis of pelvic lesions. Minim Invasive Ther Allied Technol 12: 241-244, 2003.

6. Nazim F, Hayat Z, Hannan A, Ikram U and Nazim K: Role of transvaginal ultrasound in identifying endometrial hyperplasia. J Ayub Med Coll Abbottabad 25: 100-102, 2013.

7. Menon S and Sreekumari I: Role of transvaginal ultrasound in the assessment of endometrial pathology in patients with postmenopausal bleeding. Int J Reprod Contracept Obstet Gynecol 6 : 1376-1380, 2017

8. Dadayal G, Weston M, Young A, Graham JL, Mehta K, Wilkinson N and Spencer JA: Transvaginal ultrasound (TVUS)guided biopsy is safe and effective in diagnosing peritoneal carcinomatosis and recurrent pelvic malignancy. Clin Radiol 71: 1184-1192, 2016

9. Lin SY, Xiong YH, Yun M, Liu LZ, Zheng W, Lin X, Pei XQ and Li AH: Transvaginal ultrasound-guided core needle biopsy of pelvic masses. J Ultrasound Med 37: 453-461, 2018.

10. Park JJ, Kim CK and Park BK: Ultrasound-guided transvagina core biopsy of pelvic masses: Feasibility, safety, and short-term follow-up. AJR Am J Roentgenol 206: 877-882, 2016.

11. Elkattan E, Omran E and Al Inany H: The accuracy of transvaginal ultrasound and uterine artery Doppler in the prediction of adenomyosis. Middle East Fertil Soc J 15: 73-78, 2010.

12. Tellum T, Qvigstad E, Skovholt EK and Lieng M: In vivo adenomyosis tissue sampling using a transvaginal ultrasound-guided core biopsy technique for research purposes: Safety, feasibility, and effectiveness. J Minim Invasive Gynecol: Feb 8, 2019 (Epub ahead of print).
13. Roy D, Kulkarni A, Kulkarni S, Thakur MH, Maheshwari A and Tongaonkar HB: Transrectal ultrasound-guided biopsy of recurrent cervical carcinoma. Br J Radiol 81: 902-906, 2008.

14. Giede C, Toi A, Chapman W and Rosen B: The use of transrectal ultrasound to biopsy pelvic masses in women. Gynecol Oncol 95: 552-556, 2004

15. Lee DE, Park SY, Lee SR, Jeong K and Chung HW: Diagnostic usefulness of transrectal ultrasound compared with transvaginal ultrasound assessment in young Korean women with polycystic ovary syndrome. J Menopausal Med 21: 149-154, 2015.

16. Plett SK, Poder L, Brooks RA and Morgan TA: Transvaginal ultrasound-guided biopsy of deep pelvic masses: How we do it. J Ultrasound Med 35: 1113-1122, 2016.

17. Zikan M, Fischerova D, Pinkavova I, Dundr P and Cibula D: Ultrasound-guided tru-cut biopsy of abdominal and pelvic tumors in gynecology. Ultrasound Obstet Gynecol 36: 767-772, 2010.

18. Alborzi S, Rasekhi A, Shomali Z, Madadi G, Alborzi M, Kazemi $\mathrm{M}$ and Hosseini Nohandani A: Diagnostic accuracy of magnetic resonance imaging, transvaginal, and transrectal ultrasonography in deep infiltrating endometriosis. Medicine (Baltimore) 97: e9536, 2018.

19. Fischerova D: Ultrasound scanning of the pelvis and abdomen for staging of gynecological tumors: A review. Ultrasound Obstet Gynecol 38: 246-266, 2011

20. de Groot JA, Bossuyt PM, Reitsma JB, Rutjes AW, Dendukuri N, Janssen KJ and Moons KG: Verification problems in diagnostic accuracy studies: Consequences and solutions. BMJ 343 (aug02 3): $\mathrm{d} 4770,2011$

21. Knottnerus JA, van Weel C and W Muris J: Evaluation of diagnostic procedures. In: Evidence base of clinical diagnosis. Vol 324. 2nd edition. Knottnerus JA (ed). BMJ Books, London, pp477-480, 2002.

22. Clavien PA, Barkun J, de Oliveira ML, Vauthey JN, Dindo D, Schulick RD, de Santibañes E, Pekolj J, Slankamenac K, Bassi C, et al: The Clavien-Dindo classification of surgical complications: Five-year experience. Ann Surg 250: 187-196, 2009.

23. Noreikaite J, Jones P, Fitzpatrick J, Amitharaj R, Pietropaolo A Vasdev N, Chadwick D, Somani BK and Rai BP: Fosfomycin vs. quinolone-based antibiotic prophylaxis for transrectal ultrasound-guided biopsy of the prostate: A systematic review and meta-analysis. Prostate Cancer Prostatic Dis 21: 153-160, 2018.

This work is licensed under a Creative Commons Attribution-NonCommercial-NoDerivatives 4.0 International (CC BY-NC-ND 4.0) License. 